\section{Case Reports in Oncology}

\title{
Rapid Progression of Low-Grade Cervical Dysplasia into Invasive Cancer during Natalizumab Treatment for Relapsing Remitting Multiple Sclerosis
}

\author{
King Man Wan ${ }^{a}$ Martin K. Oehler ${ }^{a, b}$ \\ aDepartment of Gynaecological Oncology, Royal Adelaide Hospital, \\ Adelaide, SA, Australia; ${ }^{b}$ Discipline of Obstetrics and Gynaecology, Adelaide Medical \\ School, University of Adelaide, Adelaide, SA, Australia
}

\section{Keywords}

Natalizumab · Relapsing remitting multiple sclerosis · Cervical cancer · Dysplasia

\begin{abstract}
A 25-year-old patient treated for relapsing remitting multiple sclerosis (RRMS) with natalizumab had a rapid progression of a low grade squamous intraepithelial lesion to a FIGO stage 1B1 squamous cell carcinoma of the uterine cervix within 2 years. She was treated with radical hysterectomy and pelvic lymphadenectomy and subsequently developed a vault recurrence within 2 years. The recurrence was treated with definitive synchronous chemo-radiotherapy and she has been disease free for 7 years. This case and existing evidence on increased risk of developing cervical dysplasia under natalizumab show that MS patients under immunosuppressive therapy require close annual cervical screening with immediate investigation of $a b-$ normal test results.

(C) 2019 The Author(s) Published by S. Karger AG, Basel
\end{abstract}




\section{Background}

Natalizumab (TysabriTM), a humanized monoclonal antibody against $\alpha 4$ integrins, is a highly effective therapy for relapsing-remitting multiple sclerosis (RRMS). Its main mechanism of action is the blockade of the binding of the $\alpha 4$ integrins to their endothelial receptors, preventing inflammatory cells from crossing the blood brain barrier and entering the central nervous system [1]. Reported side effects include infusion-related reactions, fatigue, allergic reactions, opportunistic infections and reactivation of herpes virus [2]. There have been several case reports of cervical dysplasia associated with natalizumab, potentially due to its immunosuppressive effects $[3,4]$. Here we present a patient with rapidly progressive cervical dysplasia to cervical carcinoma associated with natalizumab treatment.

\section{Case Presentation}

A 25-year-old nulliparous patient was referred to colposcopy clinic for a high-grade squamous intraepithelial lesion detected on routine cervical screening cytology in April 2009. A previous Pap smear in June 2007 had shown a low-grade lesion. The patient was suffering from RRMS since age 21 and had been commenced on natalizumab $300 \mathrm{mg}$ infusions every 4 weeks in June 2008.

At colposcopy examination the patient was asymptomatic and had a cervical biopsy taken which confirmed cervical intraepithelial lesion grade 3 . She was booked for a large loop excision of transformation zone (LLETZ) procedure.

At her LLETZ 3 months later she had an extensive warty cervical lesion which was removed with several passes. The histopathology showed a poorly differentiated invasive squamous cell carcinoma (SCC) involving the full thickness of the specimen to a depth of at least 8 $\mathrm{mm}$. A focus of perineural invasion but no definite lymphovascular space invasion was seen.

The patient underwent a radical abdominal hysterectomy, bilateral pelvic lymphadenectomy and ovarian transposition. The final specimen did not show any residual CIN or carcinoma and the lymph nodes were negative. The final diagnosis was a FIGO stage 1B1 cervical SCC and no adjuvant therapy was given.

At the time of cancer diagnosis, the patient had just completed the 12th Natalizumab infusion. The treatment was stopped and 6 months later changed to monthly methylprednisone. Her RRMS has been stable since.

Follow up consisted of 3-monthly colposcopy examinations and vault cytology which were normal for 21 months. At the 2-year checkup, the patient was diagnosed with a vaginal vault lesion which on biopsy showed recurrent cervical SCC. CT, MRI and PET imaging revealed a $5 \mathrm{~cm}$ mass at the vault without distant metastatic spread. The recurrent disease was treated with radical chemo-radiotherapy consisting of external beam pelvic radiotherapy followed by pelvic boost and concurrent weekly Cisplatin chemotherapy. Treatment was completed in November 2011.

The patient has been in full clinical remission since the chemoradiation but remains HPV positive for non-16/18 high risk subtypes in August 2018. 


\section{Discussion}

Our case highlights that cervical dysplasia can rapidly progress into cancer in a patient under natalizumab treatment. There have been a number of case reports of cervical dysplasia associated with natalizumab [3,4], however, our case to the best of our knowledge is the first report on cervical cancer.

Human papilloma virus (HPV) is responsible for most cases of cervical dysplasia and cancer. While the majority of women clear cervical HPV infection within 2 years, the immune system plays a critical role in viral clearance and immunosuppression is associated with increased risk of HPV persistence and cervical dysplasia [5].

In clinical trials natalizumab did not appear to increase the overall risk of infection or malignancy when compared to placebo. However, cases of opportunistic infections such as HPV-related uveitis have been reported [2]. Furthermore, patients under natalizumab have an increased risk of developing progressive multifocal leukoencephalopathy (PML) [6, 7]. PML is a CNS opportunistic infection caused by reactivation of a clinically latent JC polyomavirus. PML invariably occurs in the context of impaired cell-mediated immunity most frequently observed in individuals with compromised immune systems. One mechanism suggested to explain the relationship between natalizumab treatment and development of PML is that by blocking $\alpha 4$ integrin and thus decreasing lymphocyte trafficking to the brain, the normal immune surveillance in the brain is reduced, allowing the reactivation of latent viruses present in the nervous system [5]. If impaired immune surveillance in the brain following natalizumab treatment is associated with JCV reactivation, it is unlikely that such a mechanism would be specific for JCV and thus such impaired surveillance could result in the reactivation and promotion of viruses in other organs, such as HPV in the uterine cervix.

The exact mechanism by which natalizumab may accelerate progression of cervical dysplasia to cancer is unknown. Our patient rapidly progressed from cervical dysplasia to carcinoma within 2 years instead of the usual 10-15 years incubation time. Natalizumab has been associated with the development of other malignancies such as malignant melanomas [8]. In melanoma antibodies to $\alpha 4 \beta 1$ integrin inhibiting the migration of lymphocytes to sites of cutaneous inflammation and cytokine injection, as well as anti- $\alpha 4$-integrin antibodies inducing apoptosis in activated, mature lymph-node $\mathrm{T}$ cells, therefore down-regulating the immune system at the primary tumor where proposed cancer models [8]. Further research is required to determine the oncogenic link between natalizumab and cervical dysplasia and subsequent cancer development.

The optimal cervical cancer screening interval for immunocompromised women is unknown. Most guidelines recommend a shorter screening interval for immunocompromised women due to the higher incidence of cervical dysplasia in this group compared to the normal population. Screening recommendations for immunocompromised women are generally derived from recommendation for women who are HIV-positive. Currently, the World Health Organization (WHO), American College of Obstetricians and Gynecologist (ACOG) and Australian guidelines recommend HIV-positive women to be screened at 3-yearly intervals $[9,10$, 11]. However, our case demonstrates that a 3 -year screening interval is potentially too long for immunocompromised women. We therefore advocate a more intense screening protocol consisting of annual testing. Due to the lack of evidence on the best screening method for immunocompromised women (ACOG recommends cytology alone with possible reflex high-risk HPV (hrHPV) testing while WHO and Australia recommend primary hrHPV testing with or without reflex cytology for HIV positive women), combined hrHPV testing and cytology currently seems to be the safest approach. Furthermore, recommendations for screen-positive 
women differ. ACOG recommend colposcopy only if at least low-grade cytological abnormality is present even if hrHPV is positive while WHO and Australia recommend all hrHPV positive women to be referred for colposcopy. In our opinion all hrHPV-positive results and cytological abnormalities in these high-risk women should be investigated by colposcopy and potential biopsy.

\section{Statement of Ethics}

The authors have no ethical conflicts to disclose.

\section{Disclosure Statement}

The authors have no conflicts of interest to disclose.

\section{References}

1 Carlos TM, Schwartz BR, Kovach NL, Yee E, Rosa M, Osborn L, et al. Vascular cell adhesion molecule-1 mediates lymphocyte adherence to cytokine-activated cultured human endothelial cells. Blood. 1990 Sep;76(5):965-70.

2 Polman CH, O'Connor PW, Havrdova E, Hutchinson M, Kappos L, Miller DH, et al.; AFFIRM Investigators. A randomized, placebo-controlled trial of natalizumab for relapsing multiple sclerosis. N Engl J Med. 2006 Mar;354(9):899-910.

3 Rolfes L, Lokhorst B, Samijn J, van Puijenbroek E. Cervical dysplasia associated with the use of natalizumab. Neth J Med. 2013 Nov;71(9):494-5.

4 Durrieu G, Dardonville Q, Clanet M, Montastruc JL. Cervical dysplasia in a patient with multiple sclerosis treated with natalizumab. Fundam Clin Pharmacol. 2018 Jun. https://doi.org/10.1111/fcp.12394.

5 Plummer M, Schiffman M, Castle PE, Maucort-Boulch D, Wheeler CM; ALTS Group. A 2-year prospective study of human papillomavirus persistence among women with a cytological diagnosis of atypical squamous cells of undetermined significance or low-grade squamous intraepithelial lesion. J Infect Dis. 2007 Jun;195(11):1582-9.

6 Major EO, Yousry TA, Clifford DB. Pathogenesis of progressive multifocal leukoencephalopathy and risks associated with treatments for multiple sclerosis: a decade of lessons learned. Lancet Neurol. 2018 May;17(5):467-80.

7 Brown BA. Natalizumab in the treatment of multiple sclerosis. Ther Clin Risk Manag. 2009 Jun;5(3):585-94.

8 Mullen JT, Vartanian TK, Atkins MB. Melanoma complicating treatment with natalizumab for multiple sclerosis. N Engl J Med. 2008 Feb;358(6):647-8.

9 Organisation WH. WHO Guidelines WHO Guidelines for Screening and Treatment of Precancerous Lesions for Cervical Cancer Prevention.

http://apps.who.int/iris/bitstream/handle/10665/94830/9789241548694_eng.pdf Accessed September $24,2018$.

10 American College of Obstetricians and Gynecologists. ACOG Practice Bulletin: Gynecologic Care for Women and Adolescents with Human Immunodefiency Virus. The College; 2016. https://www.acog.org/ClinicalGuidance-and-Publications/Practice-Bulletins/Committee-on-Practice-Bulletins-Gynecology/GynecologicCare-for-Women-and-Adolescents-With-Human-Immunodeficiency-Virus Accessed September 24, 2018

11 Brand A, Hammond I, Panther S, et al. Screening in immune-deficient women - Cancer Guidelines Wiki; August 2018 https://wiki.cancer.org.au/australia/Clinical_question:Screening_in_immunedeficient_women. Accessed November 15,2018 